\title{
- L'eau dans le monde : la nécessité, le plaisir et les risques
}

- Water in the world : the necessity, the pleasure and the risks -

par P.H. Bourrelier

Ingénieur général des mines

\section{L'eau est indispensable à l'homme mais revêt également, là où elle est abondante, un aspect ludique. Elle peut également constituer une menace pour la vie humaine quand elle est source d'inondations. L'homme cherche à l'apprivoiser.}

Substance relativement universelle et uniforme, l'eau répond à des besoins et à des attentes profondément différentes selon les régions du monde et les personnes concernées ; pour les uns, c'est une ressource rare qu'il est vital de se procurer pour seulement survivre. Pour d'autres c'est un bien social abondant dont on attend l'épanouissement matériel, ludique ou éthique. Pour certains c'est un élément de la nature perdu par le gaspillage et la pollution. Pour d'autres c'est un produit commercial, susceptible de transactions, destiné à des activités économiques. Pour d'autres encore, c'est une source de dangers et d'affrontements périodiques avec les caprices des dieux ou de la nature.

Plus encore que pour d'autres ressources, les conditions matérielles d'accès et d'usage ne sont pas les seuls, ni sans doute, les principaux déterminants ; les comportements dépendent des représentations. Pour agir à bon escient, il faut comprendre dans chaque situation, à la fois ce qu'est la ressource en eau et ce qu'est l'eau, en tant que ressource.

Je me propose, dans les lignes qui suivent, de passer une revue rapide des voies de la connaissance qui résultent de ce constat d'extrême diversité : existe-t-il un fil directeur qui ouvre la voie à des actions communes, à des transferts technologiques, à une politique planétaire de solidarité et de responsabilité ?

Le mesurage des flux est, en toute situation, une base de connaissances fondatrice de toute action ; pourtant il n'est pas toujours fait, et il est toujours incertain, particulièrement lorsqu'il s'agit des précipitations météorologiques et des eaux souterraines. Les mesures de débit sont parmi les plus imprécises ; dans un pays comme la France, on n'a par exemple qu'une estimation grossière des prélèvements pour l'irrigation.

La même observation peut être faite pour les réserves d'eau dans le sol et le sous-sol.

La qualité de l'eau peut être repérée au moyen d'indicateurs résultant des tests physico-chimiques et biologiques; sur ce point il faut faire des choix en considération des usages présumés, mais il serait très souhaitable d'établir une base commune de référence et de comparaison. L'Agence Européenne de l'Environnement en a fait le constat pour les Etats européens qui pourtant sont relativement proches par les caractéristiques naturelles et culturelles.

Water can have various meanings : a scarse ressource, an abundant social good, an element lost by pollution and wasting, a commercial product, a source of danger. In order to better get the resource under control, its location and variability have to be studied. The strategies for exploiting the resource can then be drawn up. The water management must not be dissociated from the risk management. 
Les variations sont d'importance fondamentale puisque ce qui compte en définitive c'est la disponibilité de la ressource en un lieu et à un moment donnés. Si on a généralement une idée des variations saisonnières et interannuelles courantes, une approximation scientifiquement fondée de la variabilité, c'est-à-dire de la probabilité d'observer pendant une durée donnée un flux hors d'un intervalle limité par deux seuils de référence, fait couramment défaut.

Comprendre la variabilité climatique locale constitue donc un objectif scientifique majeur ; les progrès des méthodes d'observation et de modélisation ouvrent des espoirs d'évaluation qui seront précieux pour la gestion des risques dont il sera question plus loin : peutêtre même n'est-il pas utopique d'obtenir une précision probabiliste interannuelle qui permettrait de mettre en œuvre à temps une politique de précaution.

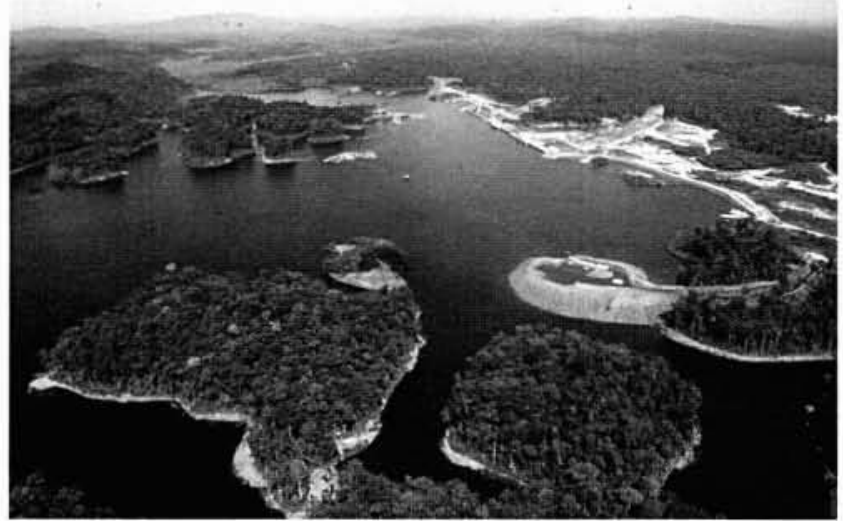

Barrage de Petit Saut en Guyane vu d'avion. (Photothèque EDF - photo Antoine Cercueil)

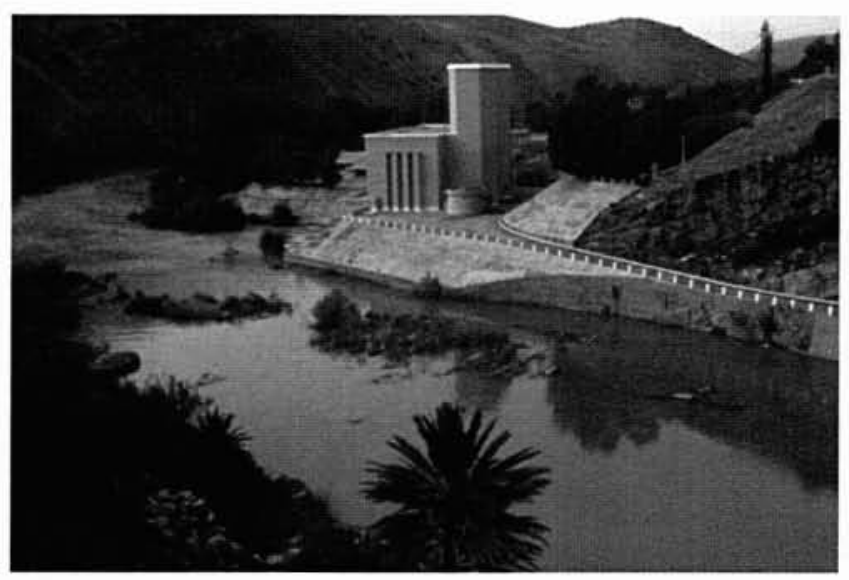

Barrage au Maroc.

(Photothèque EDF - photo Marc Morceau)

moins équilibrée, et inclure une appréciation des valeurs qui sont attachées à ces usages et qui constituent les mobiles des comportements, notamment à l'égard des eaux rejetées, dite "eaux usées", expression révélatrice.

Le mot "consommation" ne signifie en effet à peu près rien, recouvrant des réalités tout à fait disparates ; pour l'essentiel, on ne fait que mettre à profit certaines des propriétés de l'eau. Sur le plan de l'utilisation, c'est l'inventaire et l'évaluation des propriétés utilisées qu'il faudrait faire et une attention particulière devrait être portée à la comptabilité quantitative et qualitative des eaux d'irrigation et des eaux rejetées, porteuses d'un gaspillage potentiel qui est souvent pratiqué là-même où les pénuries se font sentir.

Quant aux valeurs immatérielles, c'est-à-dire aux représentations, il faut s'appuyer sur toute une gamme de recherches interdisciplinaires qui se sont dévelop-

L'effet des actions anthropiques locales n'est évalué que de façon partielle et très approximative : on saisit plus ou moins bien les prélèvements et les pollutions ainsi que les conséquences des modifications volontaires des écoulements (barrages, canaux et détournement des cours d'eau...) mais les changements de la couverture végétale et de l'usage du sol ont un effet beaucoup moins bien connu. En outre, de l'amont à l'aval d'un fleuve, seule une modélisation appropriée permet de quantifier les effets sur la variabilité du débit.

La tendance à long terme liée à la variabilité climatique habituelle et à l'effet de serre anthropique additionnel est l'objet d'une mobilisation des moyens de la recherche mondiale ; globalement l'évaporation et les précipitations devraient s'accroître et les stocks de glace se réduire. Mais au niveau local l'incertitude est complète, malgré quelques présomptions qui laissent craindre une aggravation de la situation instable des régions de transition.

\section{I ㅁ L'EAU EN TANT QUE RESSOURCE}

Comme je l'ai souligné en introduction, la connaissance des usages de l'eau devrait être exhaustive, ou pour le pées depuis des publications de Bachelard et d'Illich, les études ethnologiques sur des usages rituels des sociétés traditionnelles et les utilisations hygiénistes diffusées dans des sociétés occidentales à partir du XIXème siècle, jusqu'aux enquêtes sociologiques récentes [1].

C'est donc dans ses diverses composantes, un champ global d'investigation qu'il faut approfondir et systématiser.

\section{E LES STRATÉGIES DE RÉPONSE}

La politique de l'eau consiste à élaborer des stratégies d'exploitation et de réponse aux pressions, pour reprendre une terminologie de l'O.C.D.E. Le domaine est plus classique : il faut fixer des objectifs et des moyens qui soient cohérents. Le choix porte sur des priorités, mais aussi sur des méthodes : combinaison entre instruments règlementaires et financiers (marché, fiscalité...), structure des opérateurs et des agences publiques.

En ce qui concerne le cadre institutionnel des politiques publiques sur de tels sujets, on met actuellement de plus en plus l'accent sur l'organisation des arbitrages après débat public, et sur l'évaluation de l'efficacité des dispositifs. 
Pour illustrer les conséquences des observations qui présentent l'eau en tant que ressource, j'aborderai quatre questions :

\subsection{Biais en faveur des ouvrages lourds ou blocage des aménagements}

L'exploitation de la ressource "eau" implique évidemment des aménagements. La question qui se pose est de savoir si on n'a pas tendance à privilégier les grands investissements par rapport aux actions plus diffuses qui portent à la fois sur la ressource en eau et sur les usages. On observe certes une réticence croissante de l'opinion, fondée notamment sur la difficulté d'évaluer les impacts écologiques ; il ne faudrait pas aboutir, à l'inverse, à un blocage injustifié. La controverse sur l'opération du barrage des Trois Gorges en Chine montre combien des décisions reposent encore sur des approximations et des motivations politiques contestées. La méthodologie de consultation et de décision notamment vis-à-vis des ouvrages à fonctions multiples se cherche encore en France comme ailleurs.

\subsection{L'équilibre ressources-emplois dans l'optique du développement durable}

Les études qui ont été faites dans le cadre de I'UNESCO, ou du PLAN BLEU des pays méditerranéens mettent en évidence des perspectives de déséquilibre qui résultent de la démographie, des concepts du développement "à l'occidentale", et peut-être aussi des évolutions climatiques. Il n'y a évidemment pas de réponse simple à ces défis ; une panoplie d'actions portant sur tous les aspects peut seule être appropriée.

\subsection{Les risques et leur prévention}

La gestion des risques constitue une partie intégrante des politiques publiques de l'eau ; la seule particularité est qu'elle vise les situations d'exception, ou au moins assez aléatoires, et les crises qui s'ensuivent. Il s'agit principalement :

- des événements qui résultent de la variabilité quantitative de la ressource, soit en déficit (sécheresses) soit en excès (inondations),

- les contaminations massives, notamment bactériologiques dont la méthodologie générale d'évaluation des risques fait l'objet d'une doctrine bien établie qui repose sur le triptyque "aléa, vulnérabilité, risque".

La gestion est constituée, elle aussi, de trois composantes : gestion des crises (secours, mesures d'urgence), réparation (reconstruction, urbanisation), prévention. Il est clair que, d'une façon générale, les opérations de coopération sont plus faciles à déclencher, sous l'effet de l'émotion, pour la gestion de crise, et dans une moindre mesure pour la réparation que pour la prévention : la médiatisation croissante des événements y contribue.

Une évaluation de la politique française de prévention des risques naturels vient d'être effectuée [2] ; elle a comporté une comparaison des actions menées en France avec celles des autres pays. La réflexion et les proposi- tions faites dans ce contexte rejoignent celles qui se font jour dans le cadre européen et dans celui des Nations Unies ; à l'occasion de la décennie internationale de la prévention des catastrophes naturelles, la France a pris des initiatives intéressant les régions caraïbes et méditerranéennes.

\section{III $\square$ CONCLUSION}

Je me limiterai ici aux conclusions marquantes de l'évaluation française de la prévention des risques :

1. - Gestion de crise, réparation, prévention sont liées et doivent être étroitement associées : la prévention contribue à préparer la gestion de crise et doit s'appuyer sur le "retour d'expérience" des accidents ; il est particulièrement important que l'indemnisation et la reconstruction en tiennent largement compte. L'assurance doit être un acteur de la prévention.

2. - Toutes les parties prenantes (stake holders) doivent être impliquées dans la prévention, notamment l'Etat, les collectivités locales, les bénéficiaires de concessions ou de délégations accordées par la puissance politique, les assureurs, les aménageurs, la population.

3. - La prévention des risques naturels consiste à agir sur trois plans à la fois selon un dosage spécifique à chaque "bassin de risque" : les dispositifs de surveillance et d'alerte - la planification de l'occupation du sol et les normes de construction - les ouvrages de protection et l'entretien des milieux (lits des rivières, digues ...).

4. - S'agissant de l'eau, sécheresses et inondations sont les deux conséquences extrêmes de la variabilité météorologique ; elles ne sont pas exactement symétriques car les effets des sécheresses sont des phénomènes de durée, les crues sont des événements généralement courts et violents, mais la surveillance, l'évaluation des risques, les prévisions relèvent des mêmes coopérations interdisciplinaire entre météorologues, hydrologues, géologues, agronomes... Des progrès importants peuvent être réalisés en les associant et en utilisant les moyens de la technologie la plus performante [3].

Les variations modérées sont des phénomènes normaux, résultant naturellement des fluctuations climatiques : les crues d'ampleur courante sont utiles à l'écologie des lits des rivières, au maintien des zones humides, au rechargement des nappes, à la fertilisation. Il faut par contre se prémunir contre les effets destructeurs des phénomènes extrêmes en prévoyant leur occurrence possible, et en présence de diverses dispositions de "mitigation", d'évacuation et d'indemnisation ; les ouvrages de protection ont leur efficacité dans certaines limites, mais il faut être en mesure de faire face au risque de submersion si l'intensité de la crue dépasse le niveau de référence pour lequel ils ont été construits [4].

En résumé, à l'égard des crues comme des sécheresses, il faut mettre en cuvre des stratégies d'adaptation aux phénomènes et s'ajuster de façon efficace aux manifestations naturelles ; à l'égard d'une crue par exemple, on peut résumer la stratégie comme suit : la laisser vivre 
(en lui offrant des champs d'expansion par exemple) et vivre avec elle (en prenant les mesures pour limiter les dommages).

Cette gestion de risque doit être partagée avec la population et les collectivités ; la population apprécie de vivre auprès des rivières et du littoral, et les maires souhaitent développer des activités dans des zones susceptibles d'être affectées par des inondations : cela est à prendre en considération, mais, à condition qu'on respecte certaines limites de sécurité et qu'on applique des règles et comportements de précaution. Là encore, il est essentiel de se préoccuper de développer une attitude collective de responsabilité vis-à-vis des risques.

5. - Enfin une gestion efficace de l'eau et des risques implique une organisation des actions de prévention et de secours à des échelles pertinentes avec une répartition convenable des compétences.

Pour un fleuve comme le Rhin par exemple, on s'attache à articuler un échelon international (qui intervient sur la prévision, les dispositifs d'atténuation de crues et la coopération pour les secours), un échelon national et des échelons locaux. Ce dispositif établit des passerelles de solidarité, tout en laissant aux opérateurs locaux une grande part de responsabilité. C'est une certaine expression du principe de subsidiarité qui sert de guide à l'organisation européenne.

\section{RÉFÉRENCES}

[1] par exemple l'enquête : les citadins et l'eau. contrastes et similitudes dans le monde - laboratoire de psychologie environnementale Université Paris V. Agence de l'eau Seine-Normandie - octobre 1997.

[2] Evaluation de la politique publique de prévention des risques naturels. La documentation française - septembre 1997.

[3] La surveillance de l'environnement. Club CRIN Environnement- janvier 1998.

[4] Documents et actes des colloques de la S.H.F., Nîmes (1994). Tours (1996). Paris (1997). 